\title{
SIMULAÇÃO DE MANOBRAS ORBITAIS IMPULSIVAS COM EMPUXO LIMITADO E CONTROLE DE TRAJETÓRIA EM MALHA FECHADA
}

\author{
EVANDRO MARCONI ROCCO \\ Instituto Nacional de Pesquisas Espaciais- INPE \\ 12227-010, São José dos Campos, SP, Brasil \\ E-mail: evandro@dem.inpe.br
}

\begin{abstract}
Resumo- Objetiva-se calcular e simular manobras orbitais de um veículo espacial considerando aspectos construtivos do sistema de propulsão e controle de trajetória em malha fechada. Inicialmente consideram-se propulsores ideais capazes de aplicar empuxo de magnitude infinita, já que a variação de velocidade ocorre instantaneamente. Assim, obtêm-se as manobras impulsivas ótimas, do ponto de vista de consumo de combustível, por meio da solução do problema de Lambert (Two Point Boundary Value Problem). Então, essas manobras são simuladas considerando agora um modelo mais realista do sistema de propulsão. Devido à impossibilidade de aplicação de empuxo infinito a manobra orbital deve ser distribuída em um arco propulsivo em torno do ponto de aplicação determinado pela solução do problema de Lambert. Neste arco propulsivo utiliza-se propulsão contínua com empuxo limitado à máxima capacidade dos propulsores. Entretanto, o efeito do arco propulsivo não é exatamente igual à aplicação de um impulso, esta diferença produz um desvio da órbita final alcançada com relação à órbita de referência. Esse desvio é função da magnitude do impulso desejado, da capacidade do sistema de propulsão e das características do sistema de controle de trajetória utilizado. A avaliação desse desvio é de extrema relevância na análise de missão de veículos espaciais e no dimensionamento do sistema de controle de trajetória. Portanto neste trabalho avaliou-se como a capacidade dos propulsores e a magnitude dos impulsos influenciam nos erros da trajetória quando considerado um modelo mais realista ao invés do caso ideal representado pela abordagem impulsiva. A avaliação dos erros da trajetória torna-se ainda mais relevante se os efeitos das perturbações orbitais que atuam no veículo forem considerados. Assim, os desvios na trajetória devido às perturbações provocadas pelo termo J2 do potencial gravitacional terrestre, pelo arrasto atmosférico, pela pressão de radiação solar, pelo albedo terrestre e pelas atrações gravitacionais do Sol e da Lua foram inseridas na simulação.
\end{abstract}

Palavras-chave— Astrodinâmica, satélites artificiais, movimento orbital, manobra orbital.

\section{Introdução}

O cálculo e a simulação de manobras orbitais são de extrema relevância na astronáutica, pois somente por meio de manobras orbitais é possível posicionar adequadamente um veículo espacial de maneira que sua missão seja executada a contento. As manobras podem ser de correção e/ou de transferência. As manobras de correção ajustam a trajetória eliminando pequenos desvios causados geralmente pelas perturbações que atuam no veículo. Já as manobras de transferência alteram significativamente a órbita e são utilizadas, principalmente, durante a fase de lançamento em que o veículo é lançado em uma órbita com baixa altitude mas deve ser transferido para outra órbita de altitude mais elevada, como por exemplo, a órbita geoestacionária. Geralmente, tanto as manobras de correção quanto as de transferência devem ser realizadas de forma ótima visando minimizar o consumo de combustível, o erro no posicionamento e o tempo gasto durante a manobra. Na literatura encontram-se vários trabalhos relacionados a esse tema: Hohmann (1925), Lawden (1953), Hoelker e Silber (1959), Shternfeld (1959), Roth (1967), Gobetz e Doll (1969), Eckel (1982), Prado e Broucke (1995), Rocco (1997), Rocco et al. (2008a), Rocco et al. (2013a), entre outros.

Entretanto, a adoção de manobras impulsivas na modelagem do problema de manobrar um veículo espacial não é capaz de reproduzir a realidade. Manobras impulsivas necessitam de propulsores com capacidade de aplicação de empuxo de magnitude infinita, já que o impulso implica na alteração instantânea da velocidade. Na prática a mudança de velocidade do veículo ocorre de forma gradativa, durante um intervalo de tempo, devido à limitação da capacidade de empuxo do sistema de propulsão.

Portanto, este trabalho objetiva avaliar como a capacidade dos propulsores influencia no desvio da trajetória quando considerado um modelo mais realista ao invés do caso ideal representado pela abordagem impulsiva. A avaliação desse desvio é relevante na análise de missão de veículos espaciais e no dimensionamento do sistema de controle de trajetória.

\section{Cálculo da manobra ótima bi-impulsiva}

Antes de efetuar a simulação, a manobra biimpulsiva deve ser calculada. A determinação da manobra ótima exige a solução do problema Lambert (Two Point Boundary Value Problem). Este problema consiste em calcular a órbita de transferência que conecta um ponto na órbita inicial (posição inicial do veículo) com outro ponto na órbita final (posição final do veículo), durante um determinado intervalo de tempo $t$. As velocidades iniciais e finais na órbita de transferência, $\vec{v}_{1}$ e $\vec{v}_{2}$, para os raios vetores inicial e final, $\vec{r}_{1}$ e $\vec{r}_{2}$, são dadas por:

$$
\vec{v}_{1}=\frac{\vec{r}_{2}-f(z) \vec{r}_{1}}{g(z)}
$$




$$
\vec{v}_{2}=\frac{\dot{g}(z) \vec{r}_{2}-\vec{r}_{1}}{g(z)}
$$

Para resolver o problema as funções $f(z)$ e $g(z)$, devem ser encontradas. Este problema é conhecido como problema de Gauss ou de Lambert e suas aplicações são quase ilimitadas e incluem interceptação de satélites e manobras de rendezvous. Um estudo detalhado pode ser encontrado em Bate (1971). O algoritmo para resolver este problema por meio de variáveis universais (Battin, 1999; Bond, 1996) pode ser descrito da seguinte forma:

1- Considerando as posições inicial e final, e ainda, a direção do movimento (percurso longo ou curto), avaliar a constante $A$ em que $\Delta \theta$ é o ângulo de transferência entre os raios vetores inicial e final:

$$
\begin{gathered}
A=\operatorname{sign}(\pi-\Delta \theta) \sqrt{\left|\vec{r}_{1}\right| \vec{r}_{2} \mid(1+\cos \Delta \theta)} \\
\Delta \theta=a \cos \left(\frac{\vec{r}_{1} \cdot \vec{r}_{2}}{\left|\vec{r}_{1}\right| \vec{r}_{2} \mid}\right) \\
\operatorname{sign}(\pi-\Delta \theta)=-1 \Rightarrow \Delta \theta>\pi \\
\operatorname{sign}(\pi-\Delta \theta)=+1 \Rightarrow \Delta \theta<\pi
\end{gathered}
$$

Neste trabalho apenas o caminho mais curto foi considerado porque o objetivo é escolher a solução com o mínimo incremento de velocidade.

2- Resolva a equação (6) utilizando as equações (7 a 11$)$.

$$
\begin{gathered}
F(z)=x^{3}(z) S(z)+A \sqrt{y(z)}-t \sqrt{\mu}=0 \\
S(z)=\frac{\sqrt{z}-\sin (\sqrt{z})}{\sqrt{z^{3}}} \\
C(z)=\frac{1-\cos (\sqrt{z})}{z} \\
y(z)=\left|\vec{r}_{1}\right|+\left|\vec{r}_{2}\right|-A \frac{1-z S(z)}{\sqrt{C(z)}} \\
x(z)=\sqrt{\frac{y(z)}{C(z)}} \\
\left\{z \in R: 0 \leq z \leq(2 \pi)^{2}\right\}
\end{gathered}
$$

A função $F(z)$ é varrida por meio de um algoritmo em cascata que cobre toda a gama de soluções elípticas (Battin, 1999). A varredura é processada $n$ vezes, cada uma governada por $z$, pela tolerância na satisfação da função $F(z)$ e pelo tamanho do passo de varredura adotado. Esta abordagem permite obter soluções com boa precisão sem a necessidade da estimativa inicial das variáveis, uma vez que esta abordagem depende apenas da variável $z$ que é analisada para todo intervalo referente as soluções que utilizam órbitas elípticas.

3- Quando o método convergir avalie as funções:

$$
\begin{aligned}
& f(z)=1-\frac{y(z)}{\left|\vec{r}_{1}\right|} \\
& g(z)=A \sqrt{\frac{y(z)}{\mu}}
\end{aligned}
$$

$$
\dot{g}(z)=1-\frac{y(z)}{\left|\vec{r}_{2}\right|}
$$

4- Calcule as velocidades de transferência $\vec{v}_{1}$ e $\vec{v}_{2}$. Com as velocidades nas órbitas inicial e final, os incrementos de velocidade necessários podem ser obtidos:

$$
\begin{aligned}
& \Delta \vec{v}_{1}=\vec{v}_{1}-\vec{v}_{\text {initial }} \\
& \Delta \vec{v}_{2}=\vec{v}_{\text {final }}-\vec{v}_{2}
\end{aligned}
$$

Assim, as manobras impulsivas ótimas, do ponto de vista do consumo de combustível, são obtidas. Em seguida, essas manobras devem ser simuladas, mas agora considerando um modelo não-impulsivo para o sistema de propulsão.

\section{Simulação e controle da trajetória orbital}

O movimento orbital pode ser obtido por meio da solução da equação de Kepler (equação 16) a cada passo da simulação definida no Spacecraft Trajectory Simulator (STRS) desenvolvido por Rocco (2008b) e utilizado por Rocco et al. (2010) e Rocco (2012). Em que $M$ é a anomalia média, $u$ é a anomalia excêntrica e $e$ é a excentricidade da órbita.

$$
M=u-e \sin u
$$

Assim, dado um estado inicial e um intervalo de tempo, podemos converter o estado em elementos keplerianos resolvendo o problema inverso de posicionamento do veículo. Em seguida, utilizando a equação de Kepler esses elementos são propagados considerando um intervalo de tempo estipulado (passo da simulação). Os novos elementos keplerianos podem ser obtidos resolvendo o problema do posicionamento direto (Roy, 2005).

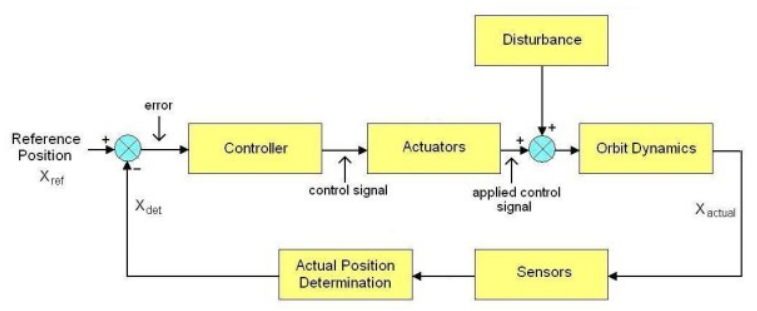

Figura 1. Arquitetura do controle de trajetória

$\mathrm{Na}$ arquitetura do simulador STRS (Fig. 1), a trajetória de referência pode ser obtida a partir de um sub-sistema de guiamento capaz de fornecer a melhor trajetória a ser seguida (solução do problema de Lambert obtida no item anterior). Esta referência é comparada continuamente com a posição atual do veículo espacial. Portanto, um sinal de erro é gerado por meio da diferença entre os estados atual e de referência. Esse sinal de erro é então enviado a um controlador proporcional integral derivativo (PID) cuja lei de controle é definida pela equação (17), em que $K_{P}, K_{I}$ e $K_{D}$ são os ganhos proporcional, integral e derivativo e $\operatorname{er}(t)$ é sinal de erro. 


$$
c(t)=K_{P} e r(t)+K_{I} \int e r(t) \mathrm{d} t+K_{D} \frac{\mathrm{d} e r(t)}{\mathrm{d} t}
$$

Usando as técnicas clássicas da teoria de controle PID, o controlador gera um sinal de controle com intuito de reduzir o erro, no regime transitório e também o erro de estado estacionário, que é enviado para os atuadores (sistema de propulsão). De acordo com o modelo do atuador é gerado um sinal de atuação que é aplicado no modelo da dinâmica do movimento, implementando as correções na posição e na velocidade do veículo. Em seguida, o estado atual é determinado de acordo com um sub-sistema que contém o modelo dos sensores, utilizado para estimar o estado considerando um sistema de coordenadas inercial centrado na Terra. Finalmente, a posição atual do veículo espacial é comparada com a posição de referência e o ciclo reinicia.

\section{Perturbações orbitais}

A avaliação dos erros da trajetória torna-se ainda mais relevante se os efeitos das perturbações orbitais que atuam no veículo forem considerados. Assim, os desvios na trajetória devido às perturbações provocadas pelo termo $\mathrm{J} 2$ do potencial gravitacional terrestre, pelo arrasto atmosférico, pela pressão de radiação solar, pelo albedo terrestre e pelas atrações gravitacionais do Sol e da Lua foram inseridas nas simulações, já que todas elas estão disponíveis no ambiente de simulação orbital STRS. Entretanto não faz parte do escopo deste trabalho a descrição detalhada da modelagem dessas perturbações. Os modelos matemáticos e alguns estudos dos efeitos das perturbações no movimento de um veículo espacial podem ser encontrados em Kaula (1966), Bate (1971), Chobotov (1991), Roy (2005), Tewari (2007), Rocco (2008c), Rocco (2010), Gomes (2011) e Kuga et al. (2011)

\section{Resultados}

Devido à impossibilidade de aplicação de um impulso de magnitude infinita a manobra orbital tem que ser distribuída em um arco propulsivo em torno da posição do impulso determinado pela solução do problema de Lambert. Neste arco propulsivo é aplicado um empuxo contínuo, limitado à capacidade máxima dos propulsores. No entanto, o efeito do arco propulsor não é exatamente equivalente à aplicação de um impulso, a diferença produz um desvio em relação à órbita final devido aos erros da magnitude $\mathrm{e}$ direção do impulso aplicado. De acordo com Chobotov (1991) o incremento de velocidade aplicado ao veículo é dado pela equação (18):

$$
\Delta V=g_{0} I_{s p} \ln \frac{m_{i}}{m_{f}}-\int_{t_{1}}^{t_{2}} g \sin \gamma \mathrm{d} t
$$

em que $g_{0}$ é a constante gravitacional ao nível do mar e $g$ na altitude do veículo, $I_{s p}$ é o impulso específico do propelente, $\gamma$ é o ângulo de voo formado entre a direção da velocidade e a direção de apontamento do propulsor, $m_{i}$ é a massa inicial do veículo e $m_{f}$ a massa final.

Da equação (18) verifica-se que a distribuição da manobra em um arco propulsivo gera uma diminuição do $\Delta V$ aplicado se a direção de aplicação do empuxo for mantida constante, já que neste caso o ângulo $\gamma$ assume valores diferentes de zero ao longo do arco propulsivo. Para minimizar esse efeito o ângulo de voo poderia ser mantido próximo de zero por meio do controle da direção de apontamento do propulsor isso garantiria que o empuxo fosse aplicado sempre na direção tangencial à trajetória. No entanto, além dessa solução ser mais complexa, pois exige atuação do sistema de controle de atitude, isso não diminui o erro na órbita final atingida pelo veículo. Para otimizar a aplicação da manobra em um arco propulsivo deve-se considerar o procedimento de otimização de manobras orbitais com propulsão contínua: Edelbaum (1961), Biggs (1978), Biggs (1979), Oliveira et al (2013). Porém a otimização de manobras com propulsão contínua também é uma tarefa difícil, já que necessita de métodos numéricos e da definição de valores iniciais para a obtenção da solução. Assim, uma possibilidade relativamente simples para minimizar o efeito causado pelo erro na direção de aplicação do empuxo seria a execução da manobra em várias etapas, cada uma delas aplicando parte do incremento de velocidade total de maneira a reduzir cada arco propulsivo e dessa forma diminuir o ângulo $\gamma$. Neste caso o erro na trajetória é minimizado mas o tempo total para atingir a órbita final é maximizado, caracterizando um problema de otimização multiobjetivo com objetivos conflitantes. O estudo de problemas multi-objetivos pode ser encontrado em Venditti et al. (2010) e Rocco et al. (2013b), mas neste trabalho não será considerado.

Para ilustrar a necessidade de quebra da manobra em vários arcos propulsivos quatro casos serão apresentados: 1- abordagem impulsiva (impulso aplicado durante 1s - Figuras 2 a 8); 2- manobra com propulsor de $500 \mathrm{~N}$ (Figuras 9 a 12); 3- manobra com propulsor de $250 \mathrm{~N}$ (Figuras 13 a 16); 4- manobra com propulsor de $50 \mathrm{~N}$ (Figuras 17 a 20).

A órbita inicial é definida pelos seguintes elementos keplerianos: $a=7000 \mathrm{~km}, e=0,0001$; $i=45^{\circ} ; \Omega=45^{\circ} ; \omega=45^{\circ}$. A órbita final é definida por: $a=7500 \mathrm{~km}, e=0,00001 ; i=45^{\circ} ; \Omega=45^{\circ}$; $\omega=45^{\circ}$. O tempo necessário para realizar a manobra de transferência e o acréscimo necessário velocidades foram determinadas pelo problema Lambert: $\mathrm{t}=3072 \mathrm{~s} ; \Delta \mathrm{v}_{1}=129 \mathrm{~m} / \mathrm{s} ; \Delta \mathrm{v}_{2}=127 \mathrm{~m} / \mathrm{s}$.

No primeiro caso foram apresentados os gráficos referentes à força perturbadora total aplicada ao veículo e ao trabalho total exercido pelas forças pertur- 
badoras (Figuras 7 e 8). Os outros casos geraram gráficos semelhantes com relação a esses parâmetros.

\subsection{Manobra impulsiva}

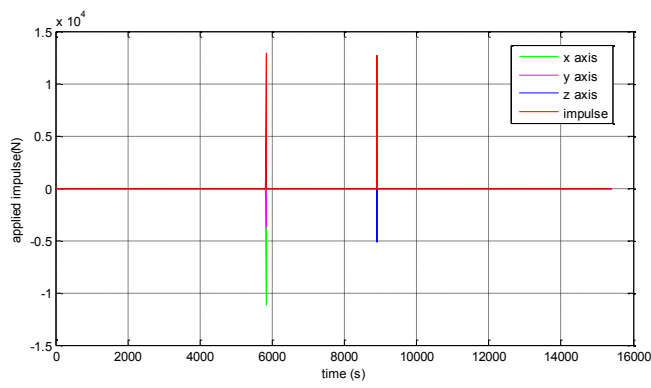

Figura 2. Empuxo aplicado

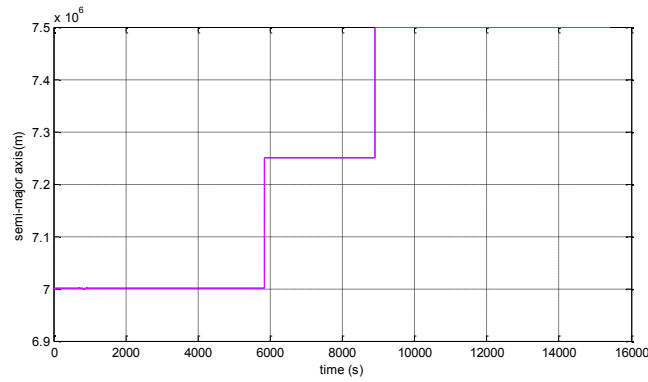

Figura 3. Semi-eixo maior sem perturbação orbital

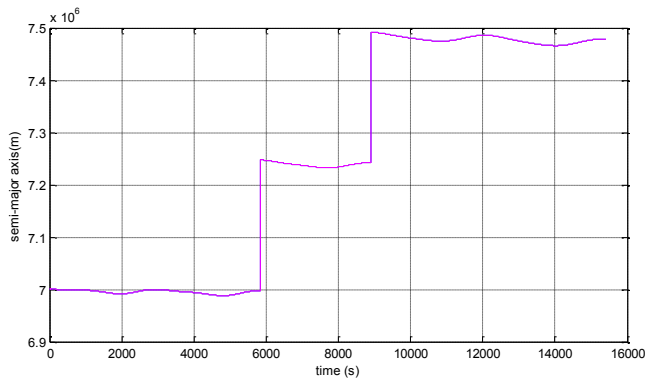

Figura 4. Semi-eixo maior com perturbação orbital

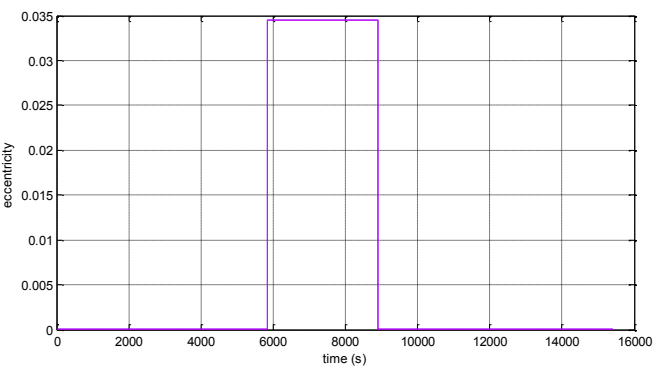

Figura 5. Excentricidade sem perturbação orbital

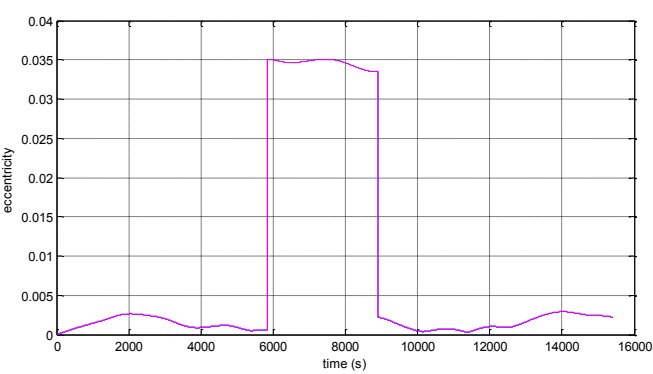

Figura 6. Excentricidade com perturbação orbital

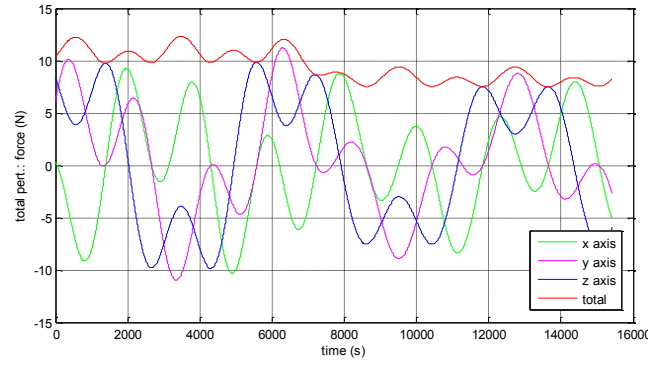

Figura 7. Força perturbadora total aplicada ao veículo

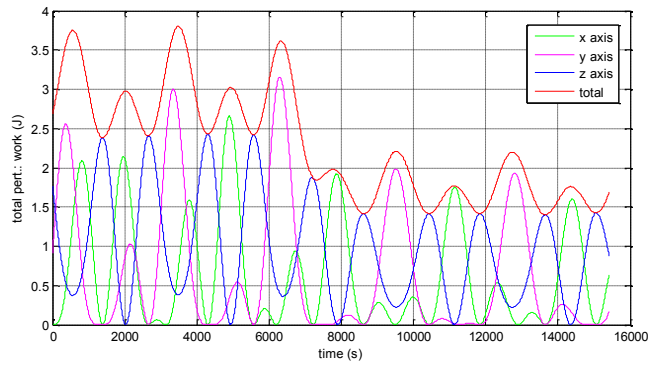

Figura 8. Trabalho total exercido pelas forças perturbadoras

\subsection{Manobra com propulsor de $500 \mathrm{~N}$}

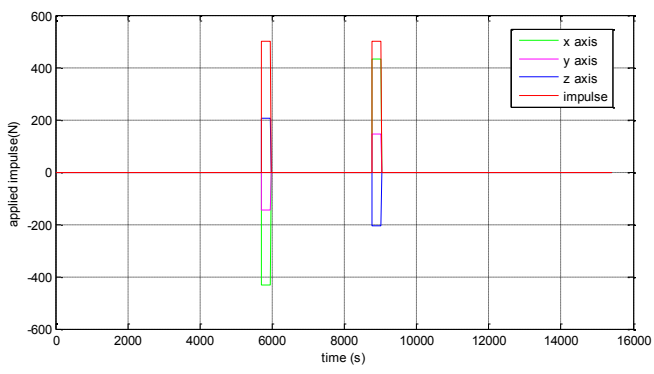

Figura 9. Empuxo aplicado ideal (500N)

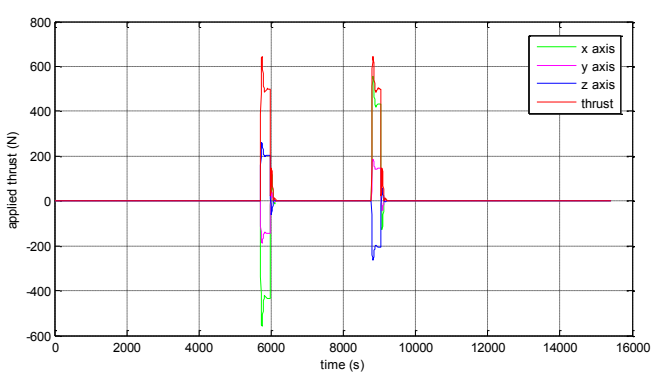

Figura 10. Empuxo aplicado $(500 \mathrm{~N})$

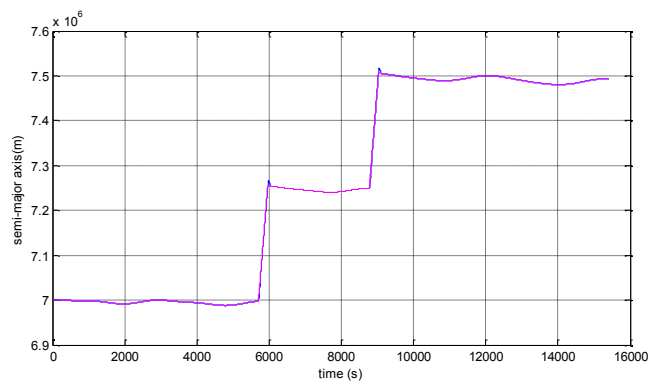

Figura 11. Semi-eixo maior com perturbação orbital (500N) 


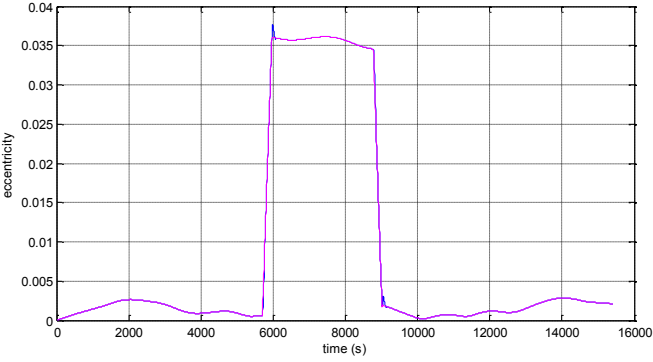

Figura 12. Excentricidade com perturbação orbital (500N)

\subsection{Manobra com propulsor de $250 \mathrm{~N}$}

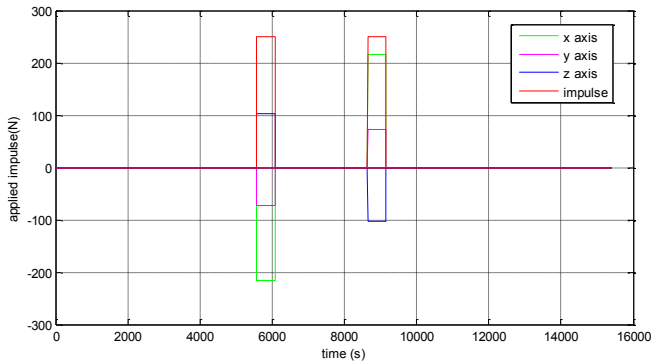

Figura 13. Empuxo aplicado ideal (250N)

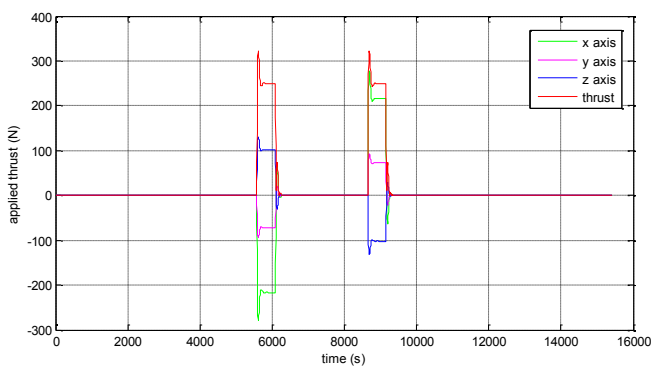

Figura 14. Empuxo aplicado(250N)

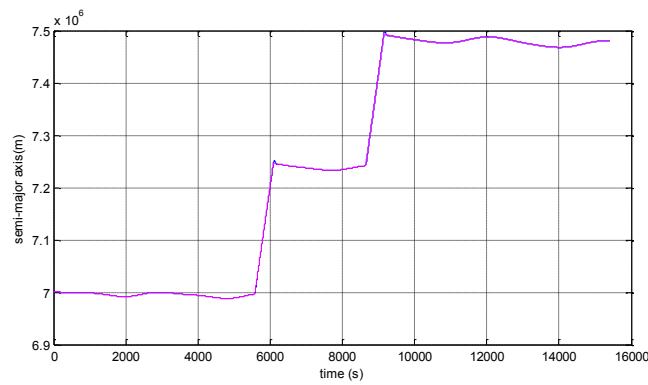

Figura 15. Semi-eixo maior com perturbação orbital $(250 \mathrm{~N})$

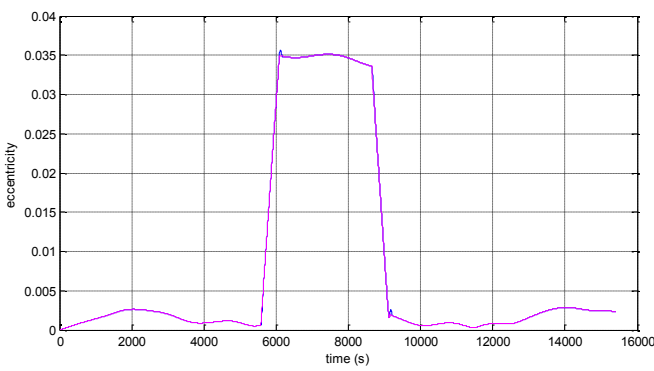

Figura 16. Excentricidade com perturbação orbital (250N)

\subsection{Manobra com propulsor de $50 \mathrm{~N}$}

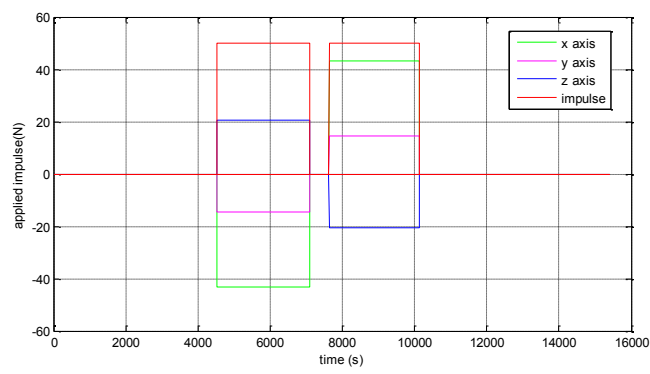

Figura 17. Empuxo aplicado ideal $(50 \mathrm{~N})$

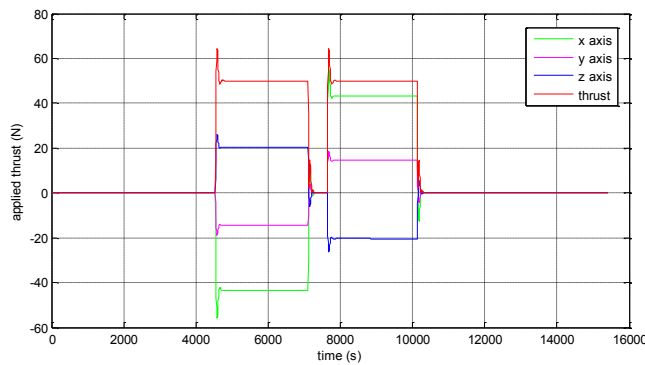

Figura 18. Empuxo aplicado (50N)

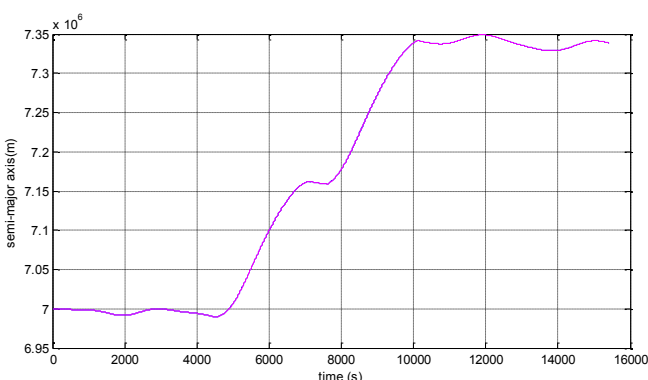

Figura 19. Semi-eixo maior com perturbação orbital $(50 \mathrm{~N})$

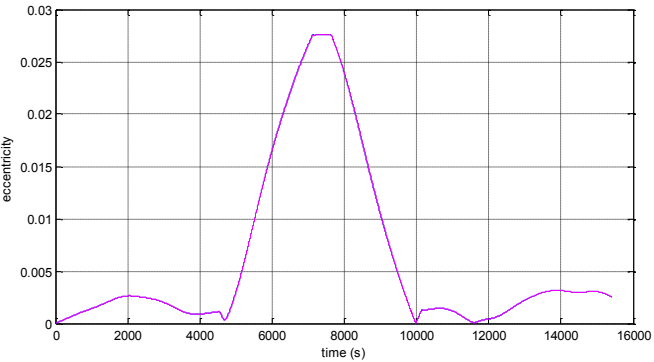

Figura 20. Excentricidade com perturbação orbital $(50 \mathrm{~N})$

\section{Conclusão}

Este trabalho representa um estudo inicial no dimensionamento do sistema de propulsão de veículos espaciais. Confirmou-se que o efeito do arco propulsivo não é exatamente equivalente à aplicação de um impulso. A diferença produz um desvio da órbita final em relação à órbita de referência. Este desvio depende da magnitude do impulso necessário para a realização da manobra, da capacidade do sistema de propulsão e das características do sistema de controle de trajetória, como verificado nos resultados. Assim, a avaliação desse desvio é relevante para a análise de uma missão espacial e no 
dimensionamento do sistema de controle de trajetória se um modelo mais realista é considerado, em vez do caso ideal representado pela abordagem impulsiva.

\section{Referências Bibliográficas}

Bate, R. R.; Mueller, D. D.; White, J. E. "Fundamentals of Astrodynamics", Dove Publications, Inc., New York, 1971.

Battin, R. H. "An Introduction to the Mathematics and Methods of astrodynamics, Rev. ed." AIAA Educational Series, Reston, 1999. DOI: $10.2514 / 4.861543$

Biggs, M.C.B., "The Optimization of Spacecraft Orbital Manoeuvres. Part I: Linearly Varying Thrust Angles", The Hatfield Polytechnic, Numerical Optimization Centre, 1978.

Biggs, M.C.B., "The Optimisation of Spacecraft Orbital Manoeuvres. Part II: Using Pontryagin's Maximum Principle”, The Hatfield Polytechnic. Numerical Optimization Centre, 1979.

Bond, V. R.; Allman, M. C. "Modern Astrodynamics: fundamentals and perturbation methods", Princeton University Press, New Jersey, 1996.

Chobotov, V.A. "Orbital Mechanics". Washington, DC: American Institute of Aeronautics and Astronautics, Inc., 365p, 1991.

Eckel, K.G., Optimal impulsive transfer with time constraint. Astronautica Acta, Vol. 9, n. 3, 1982, pp. 139-146. DOI: 10.1016/0094-5765(82)90081-9

Edelbanum, T.N., "Propulsion Requirements for Controllable Satellites" ARSJ, Aug. 1961. DOI: $10.2514 / 8.5723$

Gobetz, F.W.; Doll, J.R., 1969, “A Survey of Impulsive Trajectories", AIAA Journal, Vol. 7, No. 5: 801-834. DOI: $10.2514 / 3.5231$

Hoelker, R.F. and Silber, R., The Bi-Elliptic Transfer Between Circular Co-Planar Orbits. Tech Memo 2-59, Army Ballistic Missile Agency, Redstone Arsenal, Alabama, USA, 1959.

Hohmann, W., Die Erreichbarkeit der Himmelskorper. Oldenbourg, Munique, 1925.

Kaula, W.M. "Theory of Satellite Geodesy Applications of Satellites to Geodesyc", Blaisdell Pub. Co., 1966.

Kuga, H.K.; Carrara, V.; Kondapalli R. R. Satélites Artificiais - Movimento Orbital. INPE - São José dos Campos, 2011. 111 p. Disponível em: $<$ http://urlib.net/8JMKD3MGP7W/3ARJ3NH>.

Lawden, D.F., Minimal Rocket Trajectories. ARS Journal, Vol. 23, n. 6, 1953, pp. 360-382.

Oliveira, T.C.; Rocco, E.M.; Ferreira, J.L.; Prado, A.F.B.A.. "Minimum Fuel Low-Thrust Transfers for Satellites Using a Permanent Magnet Hall Thruster". Mathematical Problems in Engineering, v. 2013, p. 1-12, 2013. DOI: $10.1155 / 2013 / 945030$

Prado A.F.B.A. and Broucke, R.A., Transfer Orbits in Restricted Problem, Journal of Guidance Control and Dynamics, Vol. 18, n. 3, 1995, pp. 593-598. DOI: $10.2514 / 3.21428$
Rocco, E.M., Transferências Orbitais Bi-Impulsivas com Limite de Tempo. Master Thesis, (INPE6676-TDI/626, 1997.

Rocco, E.M.; Prado, A.F.B.A.; Souza, M.L.O. and Baldo, J.E., Optimal bi-impulsive non-coplanar maneuvers using hyperbolic orbital transfer with time constraint. Journal of Aerospace Engineering, Sciences and Applications, Vol. I, n. 2, 2008a, pp. 43-50.

Rocco, E.M.; Prado, A.F.B.A.; Souza, M.L.O. "Three-Dimensional Two-Impulsive Orbital Maneuvers with Time Limit" WSEAS TRANSACTIONS on APPLIED and THEORETICAL MECHANICS, Issue 1, Vol. 8, Jan. 2013a.

Rocco, E. M. Perturbed orbital motion with a PID control system for thetrajectory. In: Colóquio Brasileiro de Dinâmica Orbital, 14, Águas de Lindóia,2008. Resumos..2008b.

Rocco, E. M.; Marcelino, E.W.; Prado, A.F.B.A. "Closed Loop Control System Applied to Transfer Maneuvers Using Continuous Thrust", Mathematical Problems in Engineering, Aerospace and Sciences, São José dos Campos, Brazil, June 30 - July 3, 2010.

Rocco, E. M. Controle de trajetória com propulsão contínua para missões do tipo drag-free. In: Congresso Nacional de Engenharia Mecânica, 7, 2012, São Luís, Brasil, 2012.

Rocco, E. M. "The earth albedo model", Bremen, Germany: Center of Applied Space Technology and Microgravity (ZARM), 91 p, 2008a.

Rocco, E. M. "Evaluation of the terrestrial albedo applied to some scientific missions", Space Science Reviews, v. 151, n. 1-3, p. 135-147, 2010. DOI: $10.1007 / \mathrm{s} 11214-009-9622-6$

Rocco, E. M., Souza, M. L. O., Prado, A. B. A., "Station Keeping of Constellations Using Multiobjective Strategies", Mathematical Problems in Engineering, vol. 2013, $2013 \mathrm{~b}$. doi:10.1155/2013/476451

Roth, H.L., Minimization of the velocity increment for a bi-elliptic transfer with plane change. Astronautical Acta, Vol. 13, n. 2, 1967, pp. 119-130.

Roy, A. E. “Orbital Motion, 4th ed.” IOP Publishing Ltd, 2005.

Santos, W.G., "Simulação de Manobras Aeroassistidas de um Veículo Espacial Controlado por placas Aerodinâmicas Reguláveis e Sistema Propulsivo", (Mestrado - INPE), São José dos Campos, 2011.

Shternfeld, A., Soviet Space Science, Basic Books, Inc., New York, 1959, pp. 109-111.

Tewari, A. Atmospheric and Space Flight Dynamics: Modeling and Simulation with MATLAB and Simulink, New York, Birkhäuser Boston, 2007.

Venditti, F.C.F.; Rocco, E.M.; Prado, A.F.B.A.; Suhkanov,A. "Gravity-assisted maneuvers applied in the multi-objective optimization of interplanetary trajectories" Acta Astronautica, Elsevier Ltd., Volume 67, Issues 9-10, 2010. 